\title{
Computer Aided Facial Prosthetics Manufacturing System
}

\author{
H.K. Peng ${ }^{1, *}, M . N$. Salleh $^{1}$, and A.F. Aiman ${ }^{2}$ \\ ${ }^{1}$ School of Technology Management and Logistics, Universiti Utara Malaysia, 06010 Sintok, Kedah, \\ Malaysia \\ ${ }^{2}$ School of Manufacturing Engineering, Universiti Malaysia Perlis, 02600 Arau, Perlis, Malaysia
}

\begin{abstract}
Facial deformities can impose burden to the patient. There are many solutions for facial deformities such as plastic surgery and facial prosthetics. However, current fabrication method of facial prosthetics is high-cost and time consuming. This study aimed to identify a new method to construct a customized facial prosthetic. A 3D scanner, computer software and 3D printer were used in this study. Results showed that the new developed method can be used to produce a customized facial prosthetics. The advantages of the developed method over the conventional process are low cost, reduce waste of material and pollution in order to meet the green concept.
\end{abstract}

\section{Introduction}

Nowadays, face is important appearance for everyone. However, it is common that some people have facial deformities or disfigurement. These facial deformities or disfigurements are caused by serious accidents like burns, diseases like cancer, drug side effect or congenital facial deformities. The deformities make those people cannot live as a normal person because of their face. Their abnormal appearances make them unable to mix with the normal people or even in society. The deformities can be reduced through plastic surgery by replacing tissues from own skin. Another method is using facial prosthetics. Facial prosthetics is an artificial device that is used to alter the outward appearance of a person's face or head.

\section{Problem statement}

Current method to develop the facial prosthetics is complicated because each facial prosthetic is customized for the patient. First, a mold was made according to the affected area. The shape of the prosthetic is carefully sculpted to ensure a high quality of natural contour and fit the deformity. After the sculpture complete, a durable mold was created to

\footnotetext{
*Corresponding author: kok_peng0710@hotmail.com
} 
produce the prosthetic part. Every detail was copied to the mould to ensure it is precise and fit to the face. Due this complicated fabrication process, specialists are needed to execute the fabrication $[1,2]$. In addition, a handmade silicone ear or nose can costs up to $\$ 4000$, and requires 5 to 7 hospital visits for customization. The process takes 5-10 weeks in total [3].

Recently, 3D printing technology has emerged in additive manufacturing process for facial prosthetics fabrication. 3D printing technology has proven that it is capable of manufacturing precise and affordable facial prosthetics $[4,5]$. However, even though the cost of facial prosthetics can be reduced using the new technology, the cost of buying the technology is still out of reach for many developing countries. Most of the research mentioned above employed excessively high cost of 3D-scanning technology and 3D printing machine.

\section{Research Objectives}

The main aim of this research is to investigate a new affordable method to fabricate a customized facial prosthetics. Thus, the objectives of this research are;

I. To acquire facial area $3 \mathrm{D}$ data

II. To select the affected area and design the prosthetics.

III. Using additive manufacturing to create the prosthetics

\section{Methodology}

Method to develop the facial prosthetic can be categorized into three stages which are data acquisition, facial prosthetics 3D model development and 3d printing of facial prosthetics.

\subsection{Data acquisition}

This section discussed the method to acquire 3D data from a face.

\subsubsection{Subject Preparation}

At this stage, a human face pattern with some disruptive anatomy was used as a subject. A model constructed from Plaster of Paris (P.O.P) material was used to replicate the affected area as shown in Fig. 1.

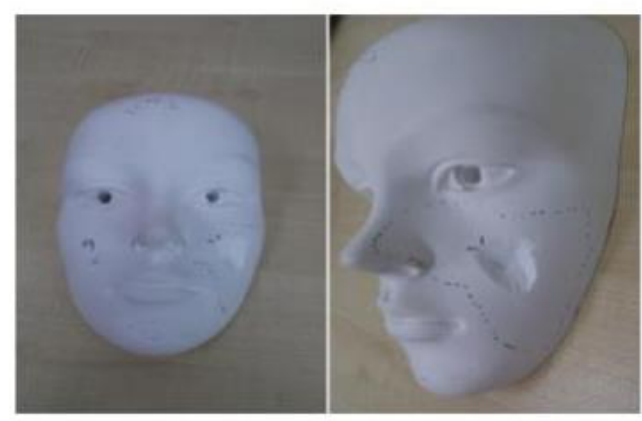

Fig. 1. Human face pattern. 


\subsubsection{Scanning Process}

Scanning process is the important stage to acquire the digital data from the subject. A Cubify Sense 3D scanner equipped with the 3D system Sense software was used to scan the subject. The result of the scanning process is shown in Fig. 2.

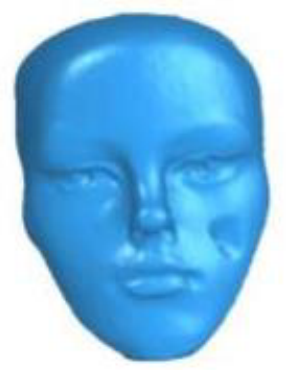

Fig. 2. Scanning result of face model.

\subsection{Facial prosthetics model development}

In this section, methods to develop the facial prosthetic model are discussed.

\subsubsection{Software preparation}

In the research, software Geomagic Sculpt developed by 3D System was used to construct the facial prosthetics due to the fast and accurate virtual sculpting software platform. This software was specifically designed for creating and modifying the 3D model that used for 3D printing.

\subsubsection{Development of facial prosthetics model}

In this section, the steps of facial prosthetics development were shown in details. The first step in this process is to import the previous $3 \mathrm{D}$ scanning result. The scanning model is imported to the Geomagic Sculpt for modifying using option. After that, curve line was created around the deformity and split. Then, subD surface is used to cover the deformity as shown in the red colour in Fig. 3. After the adjustment, the subD surface needs to be changed to become meshed and applied on the damaged face. The results of the development of facial prosthetics are perfectly smooth as shown in Fig. 4.

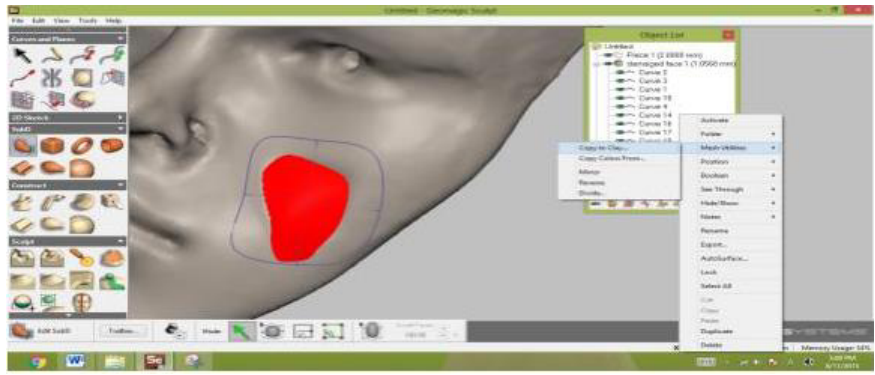

Fig. 3. The facial prosthetics model development. 


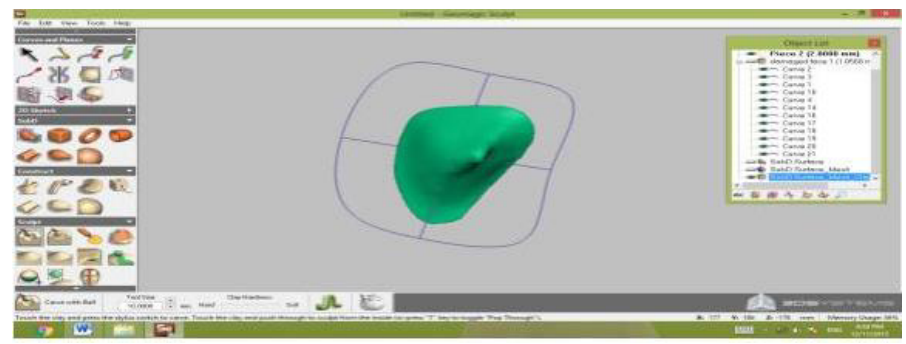

Fig. 4. 3D facial prosthetic model.

\subsubsection{Result}

Result shows the new method can be used to develop facial prosthetics. The part was fully covered the damaged face and fit the deformity shown in Fig. 5.

\subsection{D Printing Of Facial Prosthetics}

This section discussed the process of 3D printing for the facial prosthetics model.

\subsection{1 $3 D$ printer preparation}

3D printer is the most important tool in this project because it was used to fabricate the developed facial prosthetics into a tangible part. In this project, the 3D printer that was used to print out the developed facial prosthetics is Flashforge Creator Pro Dual Head 3D Printer. Material used for the facial prosthetic is Polylactid Acid (PLA).

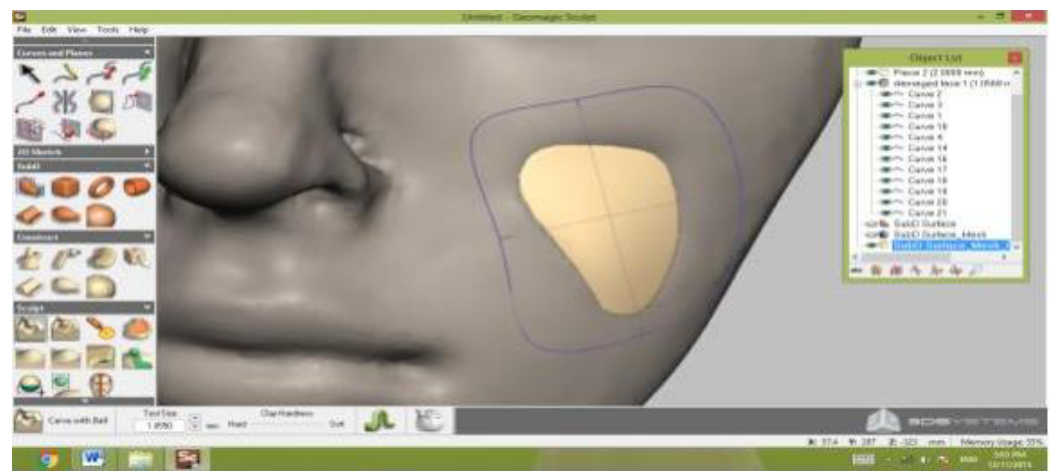

Fig. 5. 3D facial prosthetic model fits perfectly into the damaged area.

\subsubsection{D Printing Process of Facial Prosthetics}

The result from the previous 3D facial prosthetics model was exported to STereoLithography (STL) format into the MakerWare software. MakerWare can be used to set the 3D printer according to the user preference, e.g. to control the temperature of the extruder, monitor the part, adding rafting and support to make sure the part is printed in perfect condition. After the parameter setting, the print file was exported and saved in X3G format that can only be read by the 3D printer. 
The printing process took about 20 minutes for the prosthetics part. Post processing was done to eliminate the raft and support materials. The finished facial prosthetics part covers up the deformity with a good fitting as shown in Fig. 6.

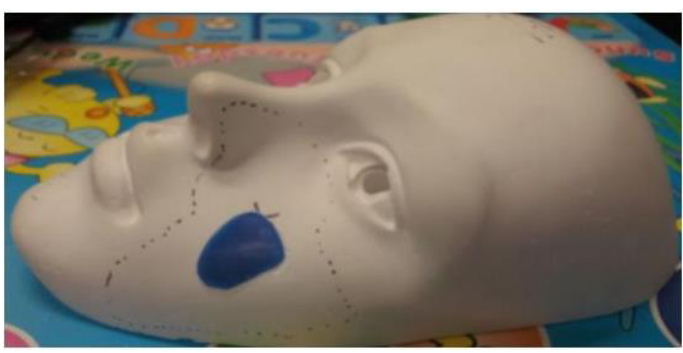

Fig. 6. Facial prosthetic fill into the damaged area.

\section{Discussion}

This project successfully identified a new method to develop the facial prosthetics using low cost $3 \mathrm{D}$ scanner and low cost $3 \mathrm{D}$ printer. The objective of this project to develop the facial prosthetics using 3D printing and 3D scanning apparatus was achieved successfully. Through this project, it brings benefit to manufacturers and doctor in term of the development of facial prosthetics for medical purposes.

The first advantage of the method is low production cost. With the low cost $3 \mathrm{D}$ scanner combined with low cost $3 \mathrm{D}$ printer, the facial prosthetics cost was reduced compared to the conventional method. The second advantage was time saving. For a professional user, they may be just consumed one day for the development of the facial prosthetics with the method in this project. For the conventional method, it takes time to finish the facial prosthetics because the process was complicated. Mostly the facial prosthetics fabrication is about one month. The third advantage of this project was the method was fully customization. There were different deformities or disfigurement in different people. So, the facial prosthetics have to fit perfectly according to patients. The fourth advantage of this method was a green process. With the use of $3 \mathrm{D}$ printing technology, there was a zero fuel emission. It reduces the air pollution. The raw material that used in this project was PLA, which is a thermoplastic and made from corn starch, sugarcane and some polymers. Therefore, the facial prosthetics were environmentally friendly and it can be recycled.

The disadvantage of the method is the level of expertise to design the facial prosthetic model. Skilled technician still in processing the 3D model was important in the process. It needed some knowledge on the function of the software so that the functions can be used in the optimum way.

\section{Conclusion}

This project has successfully identified a new low cost method to fabricate a customized facial prosthetics. The method has been explained and elaborated in the paper. For future research, researchers are suggested to apply the developed method on the real human face. 


\section{References}

1. J. Wolfaardt, A. Sugar, G. Wilkes, Int. J. Oral Maxillofac. Surg., 32, 121 (2003)

2. G.T. Grant, Aita Holmes, P.C. Liacouras, J. Garnes, W. Wilson O., J. Prosthet. Dent., 114(1), 13 (2015)

3. H. Yong, G. Xue, J. Fu, Sci. Rep., 4 (2014)

4. R. Bibb, D. Eggbeer, P. Evans, Rapid Prototyping J., 16(2), 130 (2010)

5. G.T. Grant, P. Liacouras, G.F. Santiago, J.R. Garcia, M. Al-Rakan, R. Murphy, C.R. Gordo, Ann. Plast. Surg., 72(6), 720 (2014) 